\title{
Equivalence of Mechanical and Magnetic Force in Magnetic Shape Memory Effect
}

\author{
V. KopeckÝ ${ }^{a, *}$, O. Perevertov ${ }^{a}$, L. Straka ${ }^{b}$, M. ŠevčíK ${ }^{c}$ And O. Heczko ${ }^{a}$ \\ ${ }^{a}$ Institute of Physics, ASCR, Na Slovance 2, 18221 Prague, Czech Republic \\ ${ }^{b}$ Aalto University School of Engineering, Laboratory of Engineering Materials, \\ PL 14200, FIN-00076 Aalto, Finland \\ ${ }^{c}$ Institute of Thermomechanics, ASCR, Dolejškova 5, 18200 Prague, Czech Republic
}

\begin{abstract}
High mobility of twin boundary is crucial for magnetic shape memory effect. The twin boundary can be moved by applied magnetic field or mechanical stress. In Ni-Mn-Ga $10 \mathrm{M}$ martensite there are two different, field movable, $a-c$ twin boundaries type I and II due to monoclinic lattice. For single twin boundary of both types we experimentally evaluated the equivalence of magnetic and mechanical force and the validity of generally used energy model using direct stress-strain and magnetization measurements. For type II, highly mobile twin boundary, the equivalence seems to be valid and model broadly agrees with measurement. However, for type I the calculated magnetic stress is much larger than mechanical stress needed for twin boundary motion.
\end{abstract}

DOI: 10.12693 /APhysPolA.128.754

PACS: 61.72.Mm, 75.78.Fg, 75.50.Cc, 75.70.Cn, 75.60.Ej, 62.20.D-

\section{Introduction}

Magnetic shape memory (MSM) effect is a general name for a plethora of the multiferroic effects based on interplay between ferromagnetic order and ferroelasticity [1]. One of the MSM effects is magnetically induced structure reorientation (MIR) that leads to giant deformation $(5-12 \%)$ in single phase, low-symmetry martensite $[2,3]$. Observed giant strain, in contrast to usual magnetostriction, is a result of structure reorientation by growing favourably oriented ferroelastic domain (also called variant) at the expense of other differently oriented domains. These adjacent domains are connected by twin boundary, which is moving easily and thus can be manipulated also by magnetic field [4].

The major representatives of MSM materials are compounds close to stoichiometric $\mathrm{Ni}_{2} \mathrm{MnGa}$. These alloys are also the most investigated $[1,5,6]$ as they are the most promising for applications [7-9]. In Ni-Mn-Ga alloy, parental high-temperature cubic phase transforms to various martensites $[1,10]$. For composition close to stoichiometry the martensite has $10 \mathrm{M}$ modulated structure which can be approximated by monoclinic lattice. The monoclinic structure implies the existence of type I and type II twin boundary [11, 12] that strongly differ by their twinning stress or mobility $[13,14]$.

Energetic barrier which hinders boundary movement is called twinning stress $\left(\sigma_{\mathrm{TW}}\right)$. The stress needed to move twin boundary can be induced mechanically or by magnetic field. In the simplest case the relation between magnetic and twinning stress is described by the Likhachev and Ullakko model [15]. More complex models

* corresponding author; e-mail: kopeckyv@fzu.cz were suggested, however, the simple equivalence of mechanical and magnetic force is usually retained [16, 17]. In short the model assumes that the twin boundary starts moving when the magnetic energy exceeds the mechanical barrier, which is described by relation

$$
\Delta E_{\mathrm{mag}} \geq \sigma_{\mathrm{TW}} \cdot \varepsilon_{0}
$$

where $\Delta E_{\text {mag }}$ is difference of magnetic energies of differently oriented variants in magnetic field, strain $\varepsilon_{0}=$ $1-c / a$ is given by tetragonal lattice distortion for $a \approx b$. Additionally, the model states that the magnetic energy is limited by magnitude of the magnetic anisotropy, $K_{u}$, regardless the strength of applied magnetic field $[4,15]$. This model can be used to predict existence of MIR and movement of twin boundaries in magnetic field [18, 19].

The model is just phenomenological approximation with no respect to atomic mechanism of twin boundary movement and it was shown to be broadly valid for MIR. However, the movement of individual twin boundaries has never been resolved and the model has never been tested and justified for single twin boundary. Moreover, two types of mobile twin boundaries have not been recognized until recently $[13,14,20,21]$. To test the validity of the model we directly compare magnetic twinning stress $\left(\sigma_{\mathrm{mag}}=\Delta E_{\mathrm{mag}} / \varepsilon_{0}\right)$ and mechanical stress equal to twinning stress $\left(\sigma_{\mathrm{TW}}\right)$ induced by compressive loading for single twin boundary of both types.

\section{Methods}

We used single crystal samples with composition close to stoichiometry $\mathrm{Ni}_{2} \mathrm{MnGa}$ produced by Adaptamat Ltd. Samples were rectangular shape with sizes about $20 \times 2 \times$ $1 \mathrm{~mm}^{3}$ cut approximately along $\{100\}$ planes of the parent phase. Composition of used samples is in the Table. The transformation temperature from austenite to five layered martensite (10M sometimes also $5 \mathrm{M}$ ) varied from 310 to $340 \mathrm{~K}$ as it strongly depends on composition. All experiments were done at room temperature, far enough 
from transition, i.e. in the region in which the martensitic phase is stable. The lattice parameters only slightly depend on composition and as example for one composition the lattice parameters in the monoclinic approximation are $a=0.5975 \mathrm{~nm}, b=0.5944 \mathrm{~nm}, c=0.5591 \mathrm{~nm}$, $\alpha=\beta=90^{\circ}, \gamma=90.36^{\circ}$ at room temperature.

\section{TABLE}

Comparison of magnetic and mechanical stress. Switching field $\left(\mu_{0} H_{S W}\right)$ is magnitude of magnetic field when twin boundary starts to move. $\sigma_{\text {mag }}$ is calculated magnetic stress using the model and $\sigma_{\text {mech }}$ is mechanical stress given from stress-strain curve. The stress is determined with error less than $10 \%$. Composition error is 0.5 at.\%. $R$ is a ratio of stress calculated as $R=\sigma_{\mathrm{mag}} / \sigma_{\mathrm{mech}}$.

\begin{tabular}{c|c|c|c|c|c}
\hline \hline $\begin{array}{c}\text { sample } \\
\mathrm{Ni} / \mathrm{Mn} / \mathrm{Ga}\end{array}$ & type & $\begin{array}{c}\mu_{0} H_{\mathrm{SW}} \\
{[\mathrm{T}]}\end{array}$ & $\begin{array}{c}\sigma_{\mathrm{mag}} \\
{[\mathrm{MPa}]}\end{array}$ & $\begin{array}{c}\sigma_{\text {mech }} \\
{[\mathrm{MPa}]}\end{array}$ & $R$ \\
\hline 1 & I & 0.256 & 1.572 & 0.865 & 1.82 \\
$49.8 / 29.0 / 21.2$ & II & 0.020 & 0.090 & 0.086 & 1.05 \\
2 & I & - & - & - & - \\
$49.8 / 29.0 / 21.2$ & II & 0.042 & 0.268 & 0.311 & 0.86 \\
3 & I & - & - & - & - \\
$49.8 / 29.4 / 20.8$ & II & 0.027 & 0.163 & 0.111 & 1.47 \\
4 & I & 0.354 & 1.728 & 1.110 & 1.56 \\
$50 / 28.5 / 21.5$ & II & 0.047 & 0.256 & 0.241 & 1.06
\end{tabular}

Micrographs were taken by optical microscope Zeiss using Nomarski contrast (DIC) which visualizes different tilt of surface planes by different false colors. In our case the tilt originates from various twinning. The angle between two variants connected with $a-c$ twin boundary is given approximately by $\alpha=90-2 \arctan (c / a)$. The angle $\alpha$ is usually about $3^{\circ}$ which makes twin boundary recognisable from the side of sample where $c$-axis is out of plane for one of the variants and in-plane for second variant. Different trace orientation of the boundary on the surface helps further to distinguish between type I and II twin boundaries (Fig. 1). To compress the samples

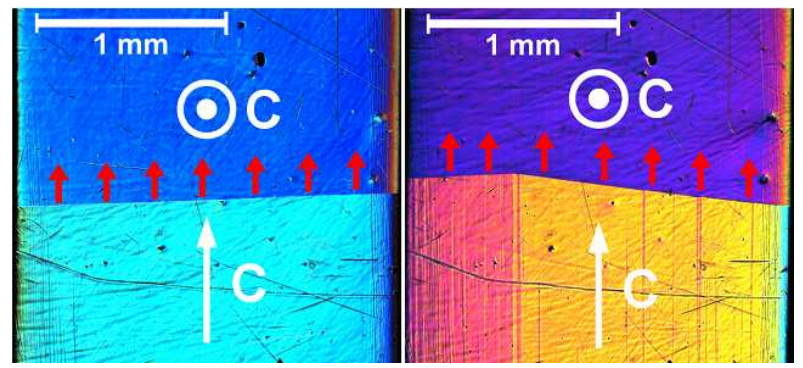

Fig. 1. Optical micrograph of twinning boundary type I (a) and type II (b) taken by optical microscope Zeiss using the Nomarski contrast (DIC). Different colours indicate different tilt of surface. Orientation of $c$-axis is marked in the figure. When stress or magnetic field along $c$-axis in plane is applied twin boundary moves as red arrows show. The boundaries are on the same sample and about same place as fiducial marks (scratches, voids) demonstrate.

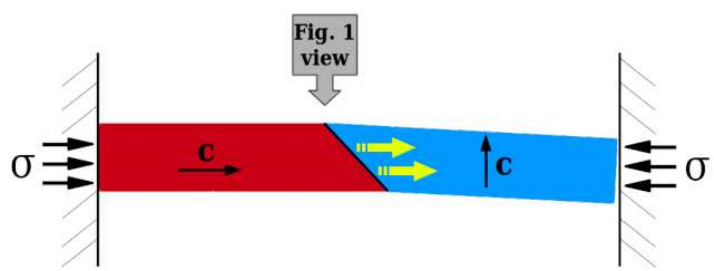

Fig. 2. Mechanical compressive stress inducing the growth of favourable variant (red) at the expense of the other one with $c$-axis perpendicular to stress by twin boundary motion (yellow arrows) resulting in large strain. The upper arrow points to the place where optical observation of twin boundary (Fig. 1) was made.

we used small custom-made stress-strain machine with step motor and linear transmission that produce continuous movement with constant rate. The sample was put between two parallel flat heads without further fixing to accommodate the large deformation due to twin boundary motion (Fig. 2). The force was measured by sensor which can be loaded up to $\pm 10 \mathrm{~N}$. The deformation strain was calculated from linear shift of the motor and initial sample's dimensions.

The magnetic force or magnetic energy was determined from the magnetization curves. These were measured by vibrating sample magnetometer (VSM) PAR with electromagnet up to $1.4 \mathrm{~T}$. From the magnetization curve of material exhibiting MIR, the field, needed to move twin boundary called switching field, can be determined. The move of twin boundary is indicated by the sharp increase of magnetization [4, 22]. From this switching field the magnetic energy, $\Delta E_{\text {mag }}$, was determined as area between magnetization curves of both variants, i.e variants with easy axis along and perpendicular to the field [4]. We neglect the effect of demagnetization as the sample was long and thus the correction is small.

Whole experiment run in several steps. We formed mechanically single twin boundary type I or type II. Using tensile stress we placed the boundary approximately $3 \mathrm{~mm}$ from the edge of the sample. Then we did compression stress-strain test. To get the boundary back to the starting position we stretched the sample again. After repeating the procedure few times the last compression run was interrupted. Without increasing forced deformation from the device the twin boundary stopped moving and stayed at the position. We removed the sample from the device and without delay installed into VSM and measured magnetization curve. As the twin boundary was not constrained, the boundary should start to move in the field which corresponds to the last value of compressive stress. After that we formed again the single twin boundary but of different type and repeated the measurement cycle.

\section{Results and discussion}

Figure 1 shows the optical micrographs of the trace of twin boundary type I and II on (100) surface. Importantly both boundaries were formed in the same sample 
and moved to approximately same position. Both twin boundaries are macrotwin boundary between twins with internal twinned structure [12-14]. The type I is straight as it runs along (101) plane but it contains $a / b$ twinning which, though, cannot be resolved in optical microscopy. In contrast, type II boundary is inclined from (101) plane by about four degrees and in addition to $a / b$ twinning it contains also monoclinic twinning. The monoclinic twinning can be readily identified in the variant with $c$-axis in plane as it forms surface relief, which is equal to twice monoclinic distortion. This twinning changes the orientation of the macrotwin boundary resulting in zigzag line of the boundary as shown in Fig. 1. In addition to much lower twinning stress, this zigzag pattern can serve as easy identification of type II twin boundary [13, 14, 23].

Figure 3 shows the typical compression test for both boundaries. Both boundaries went in the same direction through the approximately same part of the sample, but it was impossible to locate the initial position of the boundary on the exactly same place. Although the boundaries moved across the same sample the stress needed to move the boundary sharply differed and the character of the dependency varied. It is about ten times easier to move type II twin boundary than type I and the mechanical stress is in the range of tenths of MPa. Peaks in the plateau, particularly for twin boundary type I, seems to be connected to surface scratches. The observed fluctuations can be also caused by some internal inhomogeneities. For type II the connections seem to be somehow weaker as the stress-strain curve is mostly flat.

We tried to study movement of both twin boundaries by compression in each sample. Nevertheless, sometimes it was not possible to measure the stress-strain curve of twin boundary type I as the energy needed to move this boundary was larger than energy for nucleation twin boundary type II.

Several cycles of loading were done and all measured curves were roughly similar. The curves were not exactly identical as the movement of twin boundary is a stochastic process. Such behaviour was observed previously [13]. This added some uncertainty to the determination of the twinning stress and to comparison with magnetic force. After several cycles the loading was interrupted in some point and the magnetization curve with the same twin boundary was measured. The interruption points are marked in Fig. 3. From magnetization curves the switching field, i.e. field needed to move twin boundary, was determined. The magnetic energy $\Delta E_{\text {mag }}$ was calculated and then we obtained equivalent magnetic stress according to Eq. (1). The results from magnetic measurement are collected and compared with mechanical stress-strain testing in the Table. To facilitate the comparison, the ratio of magnetic and mechanic stress is also listed $\left(R=\sigma_{\mathrm{mag}} / \sigma_{\mathrm{mech}}\right)$.

The Table shows reasonable agreement for type II as the difference is mostly within experimental error. However, for type I the difference is consistently large. The magnetic force exceeds the mechanical one almost up to

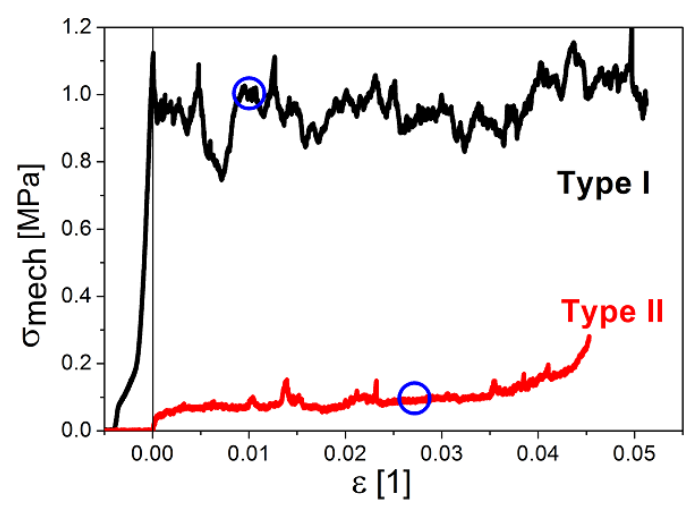

Fig. 3. Stress-strain curves of the same sample utilizing single type I and type II twin boundary movement. Evolution of compressive load affecting the sample with single twin boundary (alloy 1). Several cycles were done for each type of boundary and all of them were roughly similar. Here it is shown one of each as an example. The last cycle was interrupted for magnetic measurement at marked place (blue circle).

factor of two. It suggests that used model is invalid for type I but for disproving widely accepted model much more experiments would be needed. Additionally, the model seems to be valid for type II twin boundary. We can speculate that the model is valid and observed lower mechanical stress compared to magnetic one is due to existence shear stress originated from firm fixture of sample during the test. The forces involved are quite small and thus any additional non-axial loading might affect the measurement (see Fig. 2). In magnetic field there is no such additional force as the field is homogeneous and purely uniaxial. Moreover, the effect of magnetic domains configuration [24] and its interaction with twinning boundary is neglected.

Here, we can only conclude that the observed difference is quite puzzling. For solving this puzzle more experiments are needed which may lead to reformulation of the model. It is not helpful that the mechanism of the twin boundary motion, neither in magnetic field, nor under mechanical stress is known to great detail.

\section{Conclusion}

We studied the movement of single type I and II twin boundary resulting in structure reorientation in $\mathrm{Ni}-\mathrm{Mn}-$ Ga magnetic shape memory alloy. We were able to form both boundaries in the same samples and compared their individual behaviour. To evaluate the validity of generally used model, measured mechanical and magnetic forces needed for moving the single boundary were compared. Calculated magnetic and mechanical stress generally agreed for type II, but we registered large error for type I. This somehow puzzling observation leads to some doubt about validity of the model but more detailed measurement is needed. 


\section{Acknowledgments}

The work was funded by Czech Science Foundation grant to support excellence in research No. 14-36566G.

\section{References}

[1] O. Heczko, J. Mater. Sci. 30, 1559 (2014).

[2] K. Ullakko, J.K. Huang, C. Kanter, V.V. Kokorin, R.C. O'Handley, Appl. Phys. Lett. 69, 1966 (1996).

[3] A. Sozinov, N. Lanska, A. Soroka, W. Zou, Appl. Phys. Lett. 102, (2013).

[4] O. Heczko, N. Scheerbaum, O. Gutfleisch, in: Nanoscale Magnetic Materials and Applications, Eds. J.P. Liu, E. Fullerton, O. Gutfleisch, D.J. Sellmyer, Springer, 2009, p. 399.

[5] O. Söderberg, I. Aaltio, Y. Ge, O. Heczko, S.-P. Hannula, Mater. Sci. Eng. A-Struct. 481, 80 (2008).

[6] I. Aaltio, X.W. Liu, M. Valden, K. Lahtonen, O. Soderberg, Y. Ge, S-P. Hannula, J. Alloy Comp. 577, S367 (2013).

[7] M. Kohl, M. Gueltig, V. Pinneker, R.Z. Yin, F. Wendler, B. Krevet, Micromachines 5, 1135 (2014).

[8] B. Holz, L. Riccardi, H. Janocha, D. Naso, Adv. Eng. Mater. 14, 668 (2012).

[9] K. Schlüter, B. Holz, A. Raatz, Adv. Eng. Mater. 14, $682(2012)$.
[10] J. Pons, V.A. Chernenko, R. Santamarta, E. Cesari, Acta Mater. 48, 3027 (2000).

[11] K. Bhattacharya, Microstructure of Martensite, Oxford Univ. Press, New York 2003.

[12] H. Seiner, L. Straka, O. Heczko, J. Mech. Phys. Solids 64, 198 (2014).

[13] L. Straka, O. Heczko, H. Seiner, N. Lanska, J. Drahokoupil, A. Soroka, S. Faehler, H. Haenninen, A. Sozinov, Acta Mater. 59, 7450 (2011).

[14] O. Heczko, L. Straka, H. Seiner, Acta Mater. 61, 622 (2013).

[15] A.A. Likhachev, K. Ullakko, Phys. Lett. A 275, 142 (2000).

[16] X. Chen, Z. Moumni, Y. He, W. Zhang, J. Mech. Phys. Solids 64, 249 (2014).

[17] K. Haldar, D.C. Lagoudas, I. Karaman, J. Mech. Phys. Solids 69, 33 (2014).

[18] L. Straka, O. Heczko, H. Haenninen, Acta Mater. 56, 5492 (2008).

[19] T. Takeshita, T. Fukuda, T. Takeuchi, Mater. Sci. Eng. A-Struct. 438, 12 (2006).

[20] A. Sozinov, N. Lanska, A. Soroka, L. Straka, Appl. Phys. Lett. 99, 124103 (2011).

[21] L. Straka, H. Haenninen, O. Heczko, Appl. Phys. Lett. 98, 141902 (2011).

[22] O. Heczko, A. Sozinov, K. Ullakko, IEEE Trans. Magn. 36, 3266 (2000).

[23] R. Chulist, L. Straka, N. Lanska, A. Soroka, A. Sozinov, W. Skrotzki, Acta Mater. 61, 1913 (2013).

[24] Y.L. Ge, O. Heczko, O. Soderberg, S.P. Hannula, Scr. Mater. 54, 2155 (2006). 